\title{
A Study for the Improvement of Communications Security and Quality on Wireless Alarm Equipment
}

\author{
Masaki Fujikawa
}

\begin{abstract}
Current wireless home security system (wireless HS) has three problems: False Alarms, SIGINT, and Alarm Failure. In order to resolve these issues, it is necessary to improve the security of wireless communications and the reachability of alarm signals, as well as saving sensor's battery equivalent to the present status or less. Moreover, sharing methods to clear above problems contributes building the safe society. In line with this, the guidelines development is needed for the manufacturer and the security service companies in developing wireless HS. The aims of this paper are to determine requirements and indices of wireless communication and power consumption which are equipped with wireless HS, exemplify the solutions and verify its effectiveness, and discuss the guidelines development. The author defines two requirements and an index. Next, the author shows example solution which is based on each requirement and index, and verify its feasibility. Lastly, the author discusses the guidelines development for wireless communication and its diffusion based on the development of the clinical practice guidelines.
\end{abstract}

Index Terms-Alarm signal reachability, information security, saving battery power, wireless alarm equipment.

\section{INTRODUCTION}

\section{A. An Outline of Wireless Home Security System}

The mechanical security systems are widely introduced to offices and shops to prevent the enlargement of invasion damage. Nowadays, the Home Security System (HS) is becoming popular among households due to the rise in safety and security awareness. In particular, the wireless home security system, which connects to one controller and several sensors via radio communications, has a higher demand than the wired home security system because wireless HS can easily be installed and does not harm the appearance and atmosphere of the room.

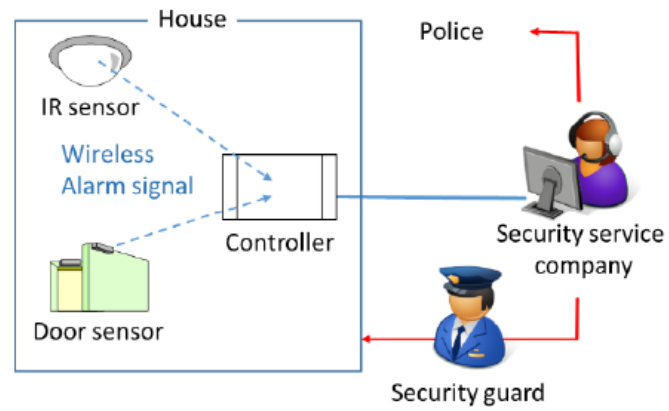

Fig. 1. Outline of the wireless home security system.

Fig. 1 shows the outline of wireless HS. Arming or

Manuscript received July 31, 2015; revise December 29, 2015.

Masaki Fujikawa is with the Security Science Institute, Tokyo, 1340092 Japan (e-mail: markie.special.agent@gmail.com). disarming the system can be done by operating the controller with a pin number. A Household member sets the system when he/she leaves from home and turns it off after returning. The sensor sends alarm signals to the controller via radio communication when it detects the intrusion-related behavior, such as when a door opens or human presence. The controller informs the incidence of intrusion to the security service company via phone line or Internet when it receives the alarm signals during the armed status. After receiving the signal, the security service firm dispatches its own security guard to the site and alerts to the police.

\section{B. Emerged Problems on Wireless HS}

In August 2014, at DEF CON 22 hacking conference, hacking results of three wireless home security systems by using the Software Defined Radio (SDR) were reported [1]. The paper pointed out the fact that the common three vulnerabilities shown below were found on the wireless communication between controller and sensor.

- No Alarms: Jamming detection functions were not enabled by default. According to this, the intruder may enter a house without being recognized by the security service company while interrupting the alarm signals by jamming attack.

- False Alarms (Replay Attack): The controller may accept the retransmission alarm signals which were sent by the sensor to it in the past. As a result, the intruder may resend the alarm signals in order to confuse the household member or/and the security service company while the status of controller is armed ${ }^{1}$.

- SIGINT: The intruder may peek the contents of alarm signals easily because these are not encrypted. Consequently, the intruder may grasp the type and the number of sensors. He/she also infers the existence of household member in house and its behavior.

In addition to these vulnerabilities, author is concerned with the occurrence of the events described below:

- Alarm Failure: Alarm signals are likely not to reach the controller if a wireless communication failure occurs between the controller and the sensor incidentally and temporarily. Collision of the alarm signals ${ }^{2}$, Electromagnetic noises [3], and Multi-pass phasing [4] are considered as the leading causes of such failure. In contrast with the case of jamming attack, these causes are likely to clear within several seconds to dozen seconds.

${ }^{1}$ In the U.S., householder should pay a fine to police when the police officers has been dispatched due to the false positive caused by sensor. In November 2014, lawsuit has piled up against the security service company due to the financial and mental damage by the false alarms [2].

${ }^{2}$ This phenomenon occurs when several sensors detect the same event and transmit alarm signals at the same time. 
Among these problems, "No Alarms" may be resolved by implementing the jamming attack detection function and the multiple radio channels in both controller and sensor by default so that interferences caused by jamming attack could be avoided. On the other hand, finding solutions for the other problems is not easy due to the electrical power problems on the side of the sensor. In general, each sensor in wireless HS is activated by a dry cell battery. In order to save battery power, (to lessen the sensor's CPU power and shorten the time of power input to the antenna to transmit alarm signal toward the controller) one-way radio communications without cryptographic protocols from sensor to controller are used. The Author is concerned that the most wireless HS with three problems are currently operated in the world.

\section{Target of This Paper}

The Author has been considering that in order to resolve three problems described earlier, it is necessary to improve the security of wireless communications and the reachability of alarm signals while reducing sensor's power consumption that is equivalent to the current consumption level or less. According to this, the author is striving to find ways to improve the quality of wireless HS.

In addition, unlike the popular wireless communication devices such as Wi-Fi routers, the author found that there are no standardized radio communication protocols for wireless HS. The vulnerability of wireless HS may have impact not only on household members but also on the infrastructure of human resources such as the employees of security service firm and the police officers. Therefore, it is desirable not to contain these three problems in wireless HS which is released in a future.

The Author believes that sharing the knowledge and technology is important and necessary to ensure the safety and the security of our society. Based on this thought, the standardized guidelines that is referred by the developers and the security service firms should be developed and diffused throughout the world. In this paper, the author creates and shows several basic solutions, verifies their effectiveness for practical use, and discusses the development and expansion of the wireless HS's guidelines.

\section{Definition of Requirement and Index}

Two Requirements and an index are defined below. The author abbreviates detail explanation of each item because its validity can be derived easily from Section I.A.

- Requirement 1: The controller can receive alarm signals from the sensor even if the communication failure occurs accidentally and temporality between the sensor and the controller.

- Requirement 2: Transmitted information between the controller and the sensor should be encrypted. The controller and the sensor can reject retransmitted information by replay attack.

- Index: Power consumption on the side of the sensor is equivalent to the current consumption level or less.

\section{E. Prerequisites}

This paper's prerequisites are stated below:

- The goal of this paper is to clear the three problems, "False Alarms", "SIGINT", and "Alarm Failure." The author does not include "No Alarms" in discussion because the controller can inform the event of jamming attack by using current jamming detection mechanism which is implemented by default.

- Accidental and temporal communication failure described in Section I.A clears within several tens of seconds.

\section{SOLUTIONS FOR REQUIREMENT 1}

In this section, the author develops and shows the basic solutions and verifies their effectiveness for practical use.

\section{A. Choice of Proper Frequency Band}

In general, radio waves are likely to increase its straightness if its frequency is increased while the diffraction decreases. Inside the house, alarm signals with high frequency tend not to reach the controller because these signals are affected by obstacles such as doors and walls. Therefore, using lower frequency is desirable in the wireless HS.

In addition, generally, a radio station license is required on each wireless equipment when it is installed and it must emit specific radio waves. For this reason, using radio frequency without a license is desirable for wireless HS because acquiring license causes financial, temporal, and personnel costs.

As of today, radio frequency band with $2.4 \mathrm{GHz}, 920 \mathrm{MHz}$, and $400 \mathrm{MHz}$ are becoming de facto standards and are widely used for digital data communication frequency band because radio station license is not required on each station. Among these bands, $2.4 \mathrm{GHz}$ band is not appropriate for wireless HS due to its higher straightness than other frequency band. (As far as the author's research, no wireless HS was found that uses $2.4 \mathrm{GHz}$ band in data communication between the sensor and the controller ${ }^{3}$ ).

Next, the author compares each frequency band from the aspect of communication distance, transmission speed, and electric consumption by using experimental results [5]. As shown in Table I, 920MHz's maximum actual communication distance is 10 times longer than that of $2.4 \mathrm{GHz}$ while its transmission speed is above 40 times higher than that of $400 \mathrm{MHz}$. In addition, communication distance from $100 \mathrm{~m}$ to $200 \mathrm{~m}$ is better for wireless HS if it is installed in a multi-story detached house or extensive grounds because inside of the house can be considered like an urbanized area with low visibility as the walls and doors are likely to block the radio waves. Moreover, the theoretical electricity consumption with $2.4 \mathrm{Kbit}$ data transmission can be estimated to $10 \mathrm{~mJ}$ for $400 \mathrm{MHz}$ band while $0.48 \mathrm{~mJ}$ for $980 \mathrm{MHz}$ band because it can transmit $2.4 \mathrm{Kbit}$ data in $1 / 41.67$ seconds.

Based on the author's research, four types of data (sensor's identifier, its serial number, and preamble and post amble data) should be included in one alarm signal, and usually, $2.4 \mathrm{Kbit}$ is enough to contain these set of information. (In fact, in the three wireless HS introduced in the DEF CON 22 Conference [1], only 9bytes of alarm signal is transmitted.) According to this, the author concludes that $920 \mathrm{MHz}$ band is

${ }^{3}$ Some of the wireless HS allow the connection between controller and the network cameras by using $2.4 \mathrm{GHz}$ band Wi-Fi. However, this paper doesn't include this type of wireless HS because network cameras require stable and enough electricity from the wall unlike the sensors author described. 
a suitable technology to be embedded in the wireless HS from the aspect of reachability, communication distance, communication speed, and electric consumption.

\section{B. Adoption of ACK Communications}

As described in Section I.B, in the current wireless HS, one-way communications from the sensor to the controller without cryptographic protocols are used in order to save the sensor's battery power. Unlike other one-way communications such as UDP (User Datagram Protocol), in wireless HS, reliability of communications is important than real-time property. When the loss of alarm signals happens, security service firm will not grasp the house's situations and will dispatch any security staff to prevent with the intrusion damage from spreading.

In addition, in the Internet technology, TCP (Transmission Control Protocol) has a scheme which retransmits data from the sender to the receiver in order to ensure the data reachability. Like this protocol, the author would like to propose a similar scheme in the wireless HS in which the sensor can retransmit alarm signals to the controller when the controller does not receive transmitted signals from the sensor. For example, the controller sends an ACK (acknowledgement) message to the sensor after receiving an alarm signal from it. In this scheme, if the sensor does not receive ACK message, the sensor can predict that the controller has not received the alarm signal and retransmits alarm signal to the controller immediately. On the other hand, even if a communication failure occurs between the sensor and the controller, the controller will receive the alarm signal as soon as possible after clearing out the earlier problem. As a result, the security service firm does not need to worry about the situation in which the controller cannot receive the alarm signals during and after the communication failure period.

Incidentally, there is a concern that the sensor's power consumption may increase due to the ACK implementation. In line with this, from the next section, the author will estimate the amount of power consumption between the $400 \mathrm{MHz}$ band with simplex communication and the $920 \mathrm{MHz}$ band with duplex communication to show the ACK installation feasibility.

1) Preparations (explanations of prerequisites and signatures)

\section{a) Prerequisites}

- The author used communication speeds of $400 \mathrm{MHz}$ and $920 \mathrm{MHz}$ band (described in Table I) to estimate sensor's power consumption.

- In Section II.B.3), the author used the consumption current of radio communication modules (see Table II) for the estimation of sensor's power consumption. Each value in Table II was derived from the previous research [6].

TABLE I: COMPARISON OF RADIO FEATURES

\begin{tabular}{l|c|c|c}
\hline \hline Frequency range & $400 \mathrm{MHz}$ & $920 \mathrm{MHz}$ & $2.4 \mathrm{GHz}$ \\
\hline Wavelength & $70 \mathrm{~cm}$ & $30 \mathrm{~cm}$ & $12 \mathrm{~cm}$ \\
\hline Transmission Power & $10 \mathrm{~mW}$ & $20 \mathrm{~mW}$ & $1 \mathrm{~mW}$ \\
\hline Antenna gain & \multicolumn{3}{|c}{$2 \mathrm{dBi}$} \\
\hline Transmission rate & $2.4 \mathrm{kbps}$ & $100 \mathrm{kbps}$ & $200 \mathrm{kbps}$ \\
\hline Receiving sensitivity & $-116 \mathrm{dBm}$ & $-106 \mathrm{dBm}$ & $-85 \mathrm{dBm}$ \\
\hline Error rate (BER) & $10^{-L}$ & $10^{-3}$ & $10^{-5}$ \\
\hline
\end{tabular}

\begin{tabular}{l|c|c|c|c}
\hline $\begin{array}{l}\text { Actual } \\
\text { distance } \\
\text { (Max.) }\end{array}$ & Downtown & $\begin{array}{c}500- \\
1000 \mathrm{~m}\end{array}$ & $\begin{array}{c}100- \\
200 \mathrm{~m}\end{array}$ & $10-50 \mathrm{~m}$ \\
\cline { 2 - 5 } & Suburb & $1-2 \mathrm{~km}$ & $\begin{array}{c}500- \\
1000 \mathrm{~m}\end{array}$ & $50-100 \mathrm{~m}$ \\
\hline \hline
\end{tabular}

TABLE II: COMPARISON OF THE CONSUMPTION CURRENT

\begin{tabular}{l|c|c}
\hline \hline Frequency range & $400 \mathrm{MHz}$ & $920 \mathrm{MHz}$ \\
\hline $\begin{array}{l}\text { Consumption current } \\
\text { (sending) }\end{array}$ & $28 \mathrm{~mA}$ & $32 \mathrm{~mA}$ \\
\hline $\begin{array}{l}\text { Consumption current } \\
\text { (receiving) }\end{array}$ & $6.2 \mathrm{~mA}$ & $15 \mathrm{~mA}$ \\
\hline \hline
\end{tabular}

\section{2) Definition of symbols}

- $T$ : Length of alarm signal. The unit of length is in bit. The same size of alarm signal is used for estimation on $400 \mathrm{MHz}$ and $920 \mathrm{MHz}$ band communication.

- $S$ : Length of ACK message. The unit of length is in bit. The Same size of ACK message is used for estimation on $400 \mathrm{MHz}$ and $920 \mathrm{MHz}$ band communication.

- Pt: Sensor's power consumption while transmitting one alarm signal. The unit is in $\mathrm{W}$ (watt). For example, if sensor uses $400 \mathrm{MHz}$ band, this symbol is used as $\operatorname{Pt}(400 \mathrm{MHz})$.

- Pr: Sensor's power consumption while receiving one ACK message. The unit is in W (watt). For example, if sensor uses $400 \mathrm{MHz}$ band, this symbol is used as $\operatorname{Pr}(400 \mathrm{MHz})$.

In this case, the sensor's power consumption $E$ in $\mathrm{J}$ (joule) in $400 \mathrm{MHz}$ and $920 \mathrm{MHz}$ are expressed in following formulas.

- If sensor uses $400 \mathrm{MHz}$ band, its power consumption for one alarm signal transmission is expressed as follows:

$$
E(400 \mathrm{MHz})=\operatorname{Pt}(400 \mathrm{MHz}) \times(T / 2,400)
$$

- If sensor uses $920 \mathrm{MHz}$ band, its power consumption for one alarm signal transmission and one ACK message receiving is expressed as follows:

$$
\begin{array}{rl}
E(920 \mathrm{MHz})=P & t(920 \mathrm{MHz}) \times(T / 100,000) \\
+ & P r(920 \mathrm{MHz}) \times(S / 100,000)
\end{array}
$$

\section{Estimation 1 (Theoretical Power Consumption)}

Hypotheses:

1) Message length of $T$ and $S$ are the same.

2) Power consumption of $P t$ and $P r$ are the same.

3) $P t(400 \mathrm{MHz})$ and $P t(920 \mathrm{MHz})$ are the same. According to this, following formula is derived:

$$
P t(400 \mathrm{MHz})=P t(920 \mathrm{MHz})=P r(920 \mathrm{MHz})=P
$$

In above hypotheses, author proves that $E(400 \mathrm{MHz})$ is larger than $E(920 \mathrm{MHz})$ by using following formula:

$$
\begin{gathered}
E(400 \mathrm{MHz})-E(920 \mathrm{MHz}) \\
=\operatorname{Pt}(400 \mathrm{MHz}) \times T / 2,400 \\
\quad-\{\operatorname{Pt}(920 \mathrm{MHz}) \times T / 100,000 \\
+\operatorname{Pr}(920 \mathrm{MHz}) \times S / 100,000\} \\
=P T / 2,400-2 P T / 100,000 \\
\cong 3.97 P T>0
\end{gathered}
$$


This formula shows $E(400 \mathrm{MHz})>E(920 \mathrm{MHz})$ and above hypotheses is true. It means that the sensor's power consumption of $920 \mathrm{MHz}$ band is lower than $400 \mathrm{MHz}$ band.

\section{Estimation 2 (Using Measured Power Consumption)}

The Author develops the formula (4) without variable replacement based on the hypotheses.

$$
\begin{gathered}
E(400 M H z)-E(920 M H z) \\
=P t(400 M H z) \times T / 2,400 \\
-\{\operatorname{Pt}(920 M H z) \times T / 100,000 \\
+\operatorname{Pr}(920 M H z) \times S / 100,000\} \\
=T[P t(400 M H z) / 2,400-\{P t(920 M H z) \\
+\operatorname{Pt}(920 M H z)\} / 100,000]
\end{gathered}
$$

If $E(400 \mathrm{MHz})-E(920 \mathrm{MHz})<0$ is set as an assumption, then the following formula is derived.

$$
\begin{gathered}
\operatorname{Pt}(400 \mathrm{MHz}) / 2,400 \\
-\{\operatorname{Pt}(920 \mathrm{MHz})+\operatorname{Pr}(920 \mathrm{MHz})\} / 100,000<0 \\
\therefore 41.7 \operatorname{Pt}(400 \mathrm{MHz})<\operatorname{Pt}(920 \mathrm{MHz})+\operatorname{Pr}(920 \mathrm{MHz})
\end{gathered}
$$

If formula (6) is true, it means that the sensor's power consumption of $920 \mathrm{MHz}$ band is higher than $400 \mathrm{MHz}$ band. However, when the values shown in Table II are input in this formula, following formula (7) is derived so that the assumption described above is not satisfied. This means that the sensor's power consumption of $920 \mathrm{MHz}$ band is lower than $400 \mathrm{MHz}$ band.

$$
41.7 \times 28 \mathrm{~mA}<32 \mathrm{~mA}+15 \mathrm{~mA}
$$

\section{E. Effectiveness Verification}

In this section, the author introduces $920 \mathrm{MHz}$ band prototype HS system with ACK message function and verifies its effectiveness compared with the $400 \mathrm{MHz}$ band HS system. Fig. 2, Fig. 3 and Table III show the prototype door sensor, the experimental environment and the specification of prototype and current sensor, respectively. Two types of sensors were attached to the point A and B shown in Fig. 3, while the steel wall and aluminum door separate between point $\mathrm{A}$ and $\mathrm{C}$. The author conducted this experiment during the day where 30 employees had been working in this environment. The Author also measured the number of door opening caused by employees and the alarm signal reception at the controller.

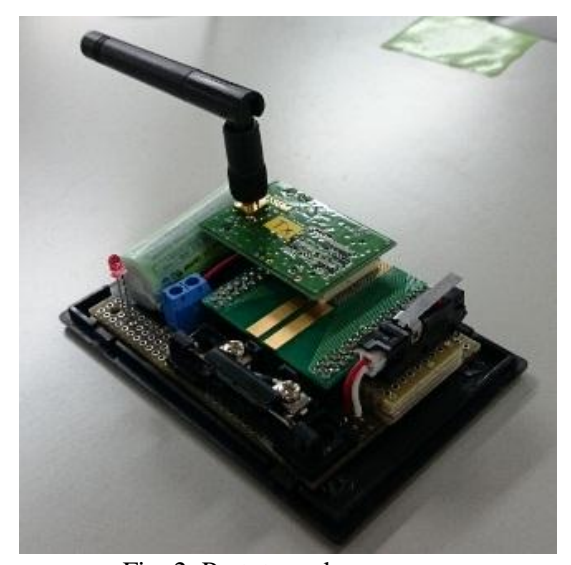

Fig. 2. Prototype door sensor.

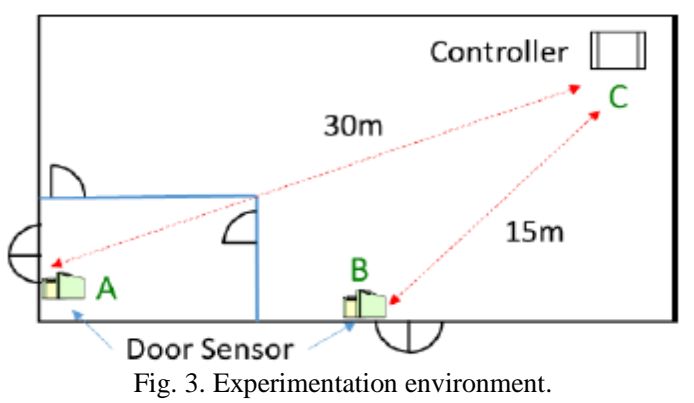

TABLE III: COMPARISON OF SPECIFICATION

\begin{tabular}{l|c|c}
\hline \hline & Current & Prototype \\
\hline Radio frequency & $426 \mathrm{MHz}$ & $920 \mathrm{MHz}$ \\
\hline Transfer speed & $1,200 \mathrm{bps}$ & $50,000 \mathrm{bps}$ \\
\hline Radio wave output & $5 \mathrm{~mW}$ & $20 \mathrm{~mW}$ \\
\hline \hline
\end{tabular}

\begin{tabular}{l|c|c}
\multicolumn{2}{c}{ TABLE IV: COMPARISON OF ALARM SIGNAL FAILURE } \\
\hline \hline Door sensor & $\begin{array}{c}400 \mathrm{MHz} \\
\text { (Current) }\end{array}$ & $\begin{array}{c}920 \mathrm{MHz} \\
\text { (Prototype) }\end{array}$ \\
\hline Point A & $40 \%(327 / 807)$ & $0 \%(0 / 807)$ \\
\hline Point B & $15 \%(49 / 3174)$ & $0 \%(0 / 3174))$ \\
\hline Completely unreached alarm signals / Total amount of alarm signals)
\end{tabular}

First, the author compared the alarm signal unreachability (See Table IV). In the current 400MHz HS system, 807 alarm signals (from point A) and 3,174 alarm signals (from point B) were sent to the controller, but 327 signals (from A) and 49 signals (from B) were unreached. On the other hand, in the prototype system, although the sensor did not receive the total amount of 40 ACK messages after sending the first alarm signals, the controller successfully received the retransmitted signals. It means that there were no completely unreached alarm signals in the prototype system.

Next, the author measured and compared the amount of electrical current on each sensor. In the current $400 \mathrm{MHz}$ sensor, $11.3 \mathrm{mAs}$ was observed while transmitting one alarm signal with $110 \mathrm{msec}$. On the other hand, in the prototype system, $0.43 \mathrm{mAs}$ was observed while sending one alarm signal with $3 \mathrm{msec}$ and receiving one ACK message with $9 \mathrm{msec}$. This means that the latter system can reduce sensor's power consumption substantially.

From these results, prototype system with $920 \mathrm{MHz}$ band with ACK communication proved the improvement of the alarm signal reachability and reduction of the power consumption.

\section{SOLUTIONS FOR REQUIREMENT 2}

In this section, the author develops and shows the basic solutions and verifies their effectiveness for practical use.

\section{A. Data Encryption}

It is a proper way to encrypt information transmitted and received by using symmetric key which is secretly shared by sensor and controller beforehand. This is because the processing time and the computation power for encryption and decryption are lower than the asymmetric key encryption (public key cryptography) [7], thus, it can reduce the sensor's power consumption. Although the stream cypher and the block cypher are present in the symmetric key encryption, the former one is more appropriate to use because it is much 
faster and can process encryption and decryption by using same electric circuit [8].

The Author focuses on the stream cypher that handles the same length of messages $M i(i=1,2, \ldots, n)$ and symmetric key $K i(i=1,2, \ldots, n)$ and outputs $E i(i=1,2, \ldots, n)$ as an encrypted message which is exclusive ORed bit by bit. $M i$ is derived by using $E i$ and $K i$ from same operation.

Unlike the block cypher, length of the message and the key should have the same size. However, according to the author's estimation, the key size will not be exceed 1,000 bits like the public key of RSA algorithm. For example, the wireless HS [1] transmits and receives only 72 bits of alarm signals. According to this, we can use a general length of symmetric key if dummy bits are added to the above signals (for example, in AES algorithm, 128 bits, 192 bits, and 256 bits of symmetric key can be used).

\section{B. Measures against the Replay Attack}

In general, using the nonce value and the keyed-hash Message Authentication Code (HMAC) is a known countermeasure against the reply attack. The reasons why using nonce and HMAC is effective are shown below:

- Using timestamp is an option instead of nonce value, however, it is necessary to embed an internal clock inside the sensor and synchronize both clocks between on the controller and on the sensor securely.

- The Author already proposes the adaptation of ACK communication described in Section II.B. Taking advantage of this communication, the controller can transmit nonce value to the sensor. Each sensor can confirm the authenticity of nonce value if HMAC is used in this communication.

The controller and the sensor can reject the retransmitted information and confirm the authenticity of the information received if the nonce value and the HMAC are included in each data.

\section{Communications Protocol 1 (Transmission of Alarm Signal and ACK Message)}

Fig. 4 shows the basic protocol in transmitting one alarm signal and one ACK message. The Sensor and the controller secretly share key as symmetric key, challenge chg_$x$ as nonce value, and seed seed for pseudo-random number generation.

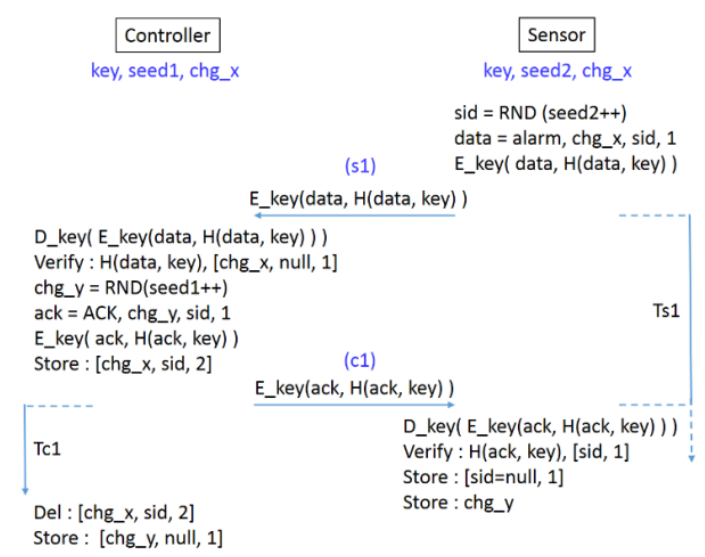

Fig. 4. Communications protocol 1.

The sensor generates session number sid randomly when it transmits alarm signal. In this case, sid doubles as the nonce value for the controller. Next, the sensor calculates HMAC of the data with the number of alarm transmission (=1), encrypts data HMAC by using key, and sends them to the controller. After that, the sensor waits to receive the ACK message up to Ts 1 seconds.

The controller decrypts the alarm signal "s1" by using key and confirms the accordance of HMAC. Next, the controller verifies the content of "s 1 ". In this case, the controller notices two facts, that "s1" is not a retransmitted alarm signal with the situation of "Alarm Failure" or "ACK Failure" and "s1" is not a retransmitted alarm signal sent by replay attack assailant because (1) received $\operatorname{chg}_{-} x$ is the same as the controller's $c h g \_x$, (2) value of sid is null (it means the controller didn't receive any sid before), and (3) the number of alarm transmission is less than or equal to one (it means this is a newly received alarm signal).

The controller randomly generates a nonce value chg_y $y$ for the sensor. Next, the controller calculates HMAC of ack with the number of ACK transmission (=1), encrypts ack and HMAC by using key, and sends them to the sensor. After, the controller saves $c h g \_x$, sid, and 2 temporarily in order to reject the replay attack with "s1" and prepare the transmission failure of "c1." These data are deleted after $T c 1$ seconds ( $T c 1$ is set longer than $T s 1$ ). If the controller does not receive retransmitted alarm signal from the sensor within TC1 seconds (it means the ACK Failure did not occur), the controller saves the set of $c h g_{-} y$, sid (=null), and the number of ACK transmission (=1) as a proof of above situation.

If the sensor receives information within $T s 1$ seconds, the sensor confirms it as an ACK message and decrypts. After the confirmation of HMAC accordance, the sensor checks that "c1" is not a retransmitted information from a replay attack assailant. This situation is assumed from two reasons: (1) the received sid is the same with the sid which the sensor has and (2) the number of ACK transmission is less than or equal to one (it means the newly received ACK signal). After that, sensor saves $\mathrm{chg}_{-} y$ for the next alarm signal transmission as a nonce value. It also saves sid (=null) and the number of transmission $(=1)$ in order to reject the replay attack by using "c1."

\section{Communications Protocol 2 (In Case of Alarm Failure)}

In this session part, the sensor's and the controller's behavior are shown when the alarm signal "s1" does not reach the controller (see Fig. 5 and refer Section III.C).

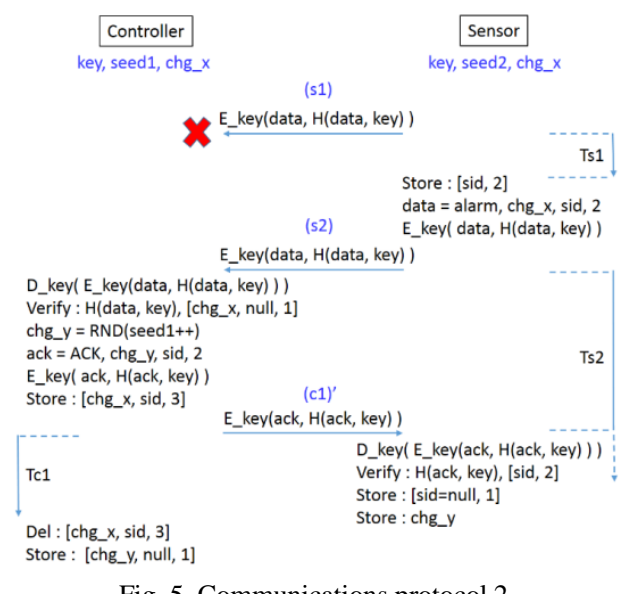

Fig. 5. Communications protocol 2. 
If the sensor does not receive the ACK message regarding "s1" within Ts1 seconds, the sensor holds the sid and the number of alarm signal transmission (=2) in order to reject/ignore "c1" which was planned to receive by the sensor. Next, the sensor transmits alarm signal "s2" as a retransmission which includes the number of alarm signal transmission $(=2)$.

The controller confirms the correspondence of HMAC which is included in "s2" and the fact that it is not a retransmitted alarm signal from a replay attack assailant. In this case, the controller notices that "s2" is a retransmitted alarm signal generated by the sensor as "Alarm Failure" because (1) the received $\operatorname{chg}_{-} x$ is the same with the controller's $\operatorname{chg}_{-} x$, (2) the value of sid is null (it means the controller didn't receive any sid before), and (3) the number of alarm transmission is more than one (it means this is not a newly received alarm signal).

The controller sets the number of ACK transmission (=2) and sends the ACK message $\mathrm{cl}$ ' in the same manner as Section III.C. Then, the controller saves the set of $\mathrm{chg} \__{-} x$, sid, and the number of ACK transmission (=3) to reject/ignore "s1" and "s2" with replay attack and prepare for the ACK Failure of c1'. The controller should set the standby time $T c 1$ longer than $T s 2$ (=Ts1).

If the sensor receives information within Ts2 seconds, sensor assures it as an ACK message and decrypts. The sensor notices that the received information is not a retransmitted one from replay attack assailant because (1) the received sid is the same with the sensor's sid and (2) the number of ACK message transmission is lower than or equal to two. After that, sensor stores sid (=null) and the number of alarm signal transmission $(=1)$ in order to prevent a replay attack by using c1'. The Sensor also saves $\operatorname{chg}_{-} y$ as a challenge for the next alarm signal transmission.

\section{E. Communications Protocol 3 (In Case of ACK Failure)}

In this session part, the sensor's and the controller's behavior are shown when the ACK message "c1" does not reach the sensor (see Fig. 6 and refer the Section III.C).

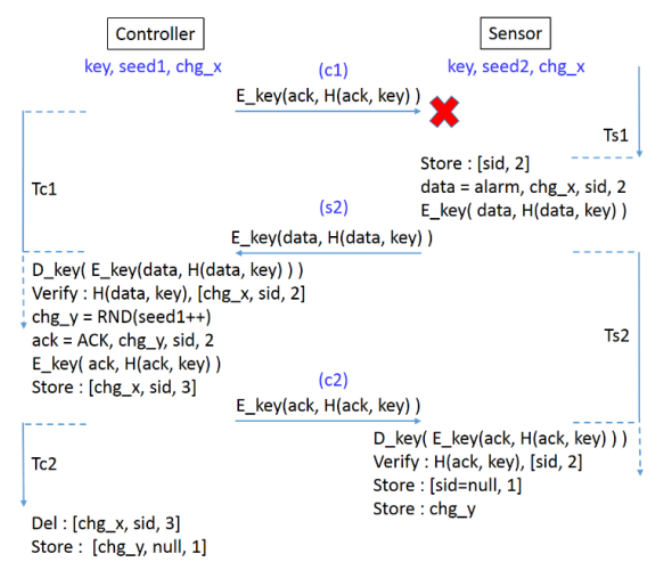

Fig. 6. Communications protocol 3.

If the sensor does not receive the ACK message "c1" regarding "s1" within Ts1 seconds, the sensor holds sid and the number of alarm signal transmission (=2) in order to reject/ignore "cl" which is expected to receive by the sensor. Next, the sensor transmits the alarm signal "s2" which includes the number of alarm signal transmission (=2).
The controller confirms the correspondence of HMAC which is included in " 2 " " and the fact that it is not a retransmitted alarm signal from a replay attack assailant. In this case, the controller notices that "s2" is a retransmitted alarm signal generated by the sensor with the situation of "ACK Failure" because (1) the received $\operatorname{chg}_{-} x$ and sid are the same with the controller's one and (2) the number of alarm transmissions is more than one (it means this is not a newly received alarm signal).

The controller sets the number of ACK transmission (=2) and sends the ACK message "c2" in the same manner as Section III.C. After that, the controller saves the set of $\operatorname{chg}_{-} y$, sid, and the number of ACK transmissions (=3) to reject/ignore "s1" and "s2" with replay attack and prepare for the ACK Failure of "c2". The controller should set the standby time $T c 2$ (=Tc1) longer than $T s 2$ (=Ts1).

If the sensor receives the information within Ts2 seconds, sensor confirms that it as an ACK message and decrypts. The sensor notices that the received information is not a retransmitted one from replay attack assailant because (1) the received sid is the same with the sensor's sid and (2) the number of ACK message transmission is less than or equal to two. After that, the sensor stores sid (=null) and the number of alarm signal transmissions $(=1)$ in order to prevent from the replay attack by using "c1" and "c2." The sensor also saves $\operatorname{chg} y$ as a nonce value for the next alarm signal transmission.

\section{F. Effectiveness Verification}

In the communication protocols described in Sections III.C to III.E, all information transmitted between the sensor and the controller are encrypted by a symmetric key which is secretly shared beforehand. As a result, the information will not be seen in a conventional way.

In these protocols, both the sensor and the controller can confirm the received information whether it is from a replay attack assailant or not. Specifically, the controller checks HMAC, nonce value, session id, and the number of alarm signal transmissions which are included in one alarm signal while the sensor verifies HMAC, session id (=nonce value), and the number of ACK transmissions which are contained in one ACK message. Both entity reject/ignore the received information as a retransmitted message caused by replay attack if the received nonce value and the number of transmission is inconsistent. It is difficult for the attacker to counterfeit or alter the above data because these information with HMAC are encrypted.

As described above, illustrated protocols in this paper seems to satisfy the requirement 2 . The Author will measure the sensor's electrical current consumption after the implementation of these protocols.

\section{G. Replacement of Cryptographic Algorisms}

The Illustrated communication protocols in this paper use three types of cryptographic algorisms: Symmetric cryptography, pseudo-random number generator, and one-way hash function. These algorisms' security are not guaranteed permanently, so that each algorism should be replaced when its vulnerability is discovered. Several years are set as the period of algorism replacement. For example, NIST (National Institute of Standards and Technology) set a 
plan in 2005 to replace the prevailing algorisms (Triple DES and SHA-1) of those days with a new one until the end of 2010 [9].

In the wireless HS, each sensor is activated by a dry cell battery and it should notify the detected information to the controller immediately. Hence, it is not appropriate for the sensor to consume electrical power for the algorism replacement. Thus, the author proposes another way in which household members or employees of security service firms replaces a ROM (Read Only Memory) which is inside the sensor while the controller is set to disarmed position. In this case, two prerequisites are needed: (1) The ROM can detach easily like the Compact Flash Memory Card; (2) The ROM contains three algorisms beforehand;

In fact, in Japan, a security firm's employee checks the wireless HS once in several years while the U.S.' household member installs the wireless HS by themselves. For these reasons, the proposed method for algorisms replacement seems to be a proper and feasible solution.

\section{GUIDELINES DEVELOPMENT AND ITS EXPANSION}

In this section, guidelines development and its expansion are discussed in order to promote sharing and referring the knowledge between the manufacturer and the security service firm. These guidelines feature the quality improvement for the wireless HS.

In the medical fields, "evidence-based treatment [10]" is standardized internationally and the guidelines based on this thought are referred and used [11]-[13]. Guidelines show the recommended, unbiased, and the best treatment methods based on the latest clinical evidence. Guidelines are developed through six stages (Preparation, Scope, Systematic review, Recommendation, Finalization, and Updating) and is open to the public.

The author thought that the guidelines development method in treatment is a good example in order to develop the guidelines with unbiased and reliable technologies for the wireless HS. From the following section, the author's considerations are described for the development of the wireless HS guidelines.

\section{A. Preparations}

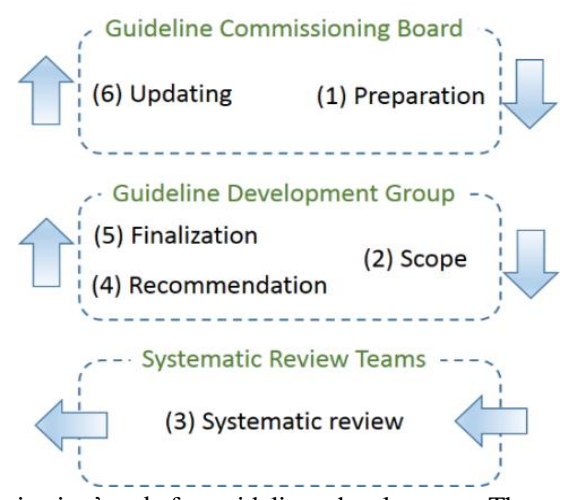

Fig. 7. Organization's role for guidelines development. There are six stages to develop medical guidelines.

\section{1) In medical guidelines}

As shown in Fig. 7, guidelines are developed by three organizations. In particular, the existence of the Guideline
Development Group and the Systematic Review Team are important. The former's role are planning the development of guidelines and making the drafts as a brain while the latter's role is doing the systematic review as a taskforce based on professional expertise.

\section{2) In wireless $H S$ guidelines}

For example, in Japan, each security service firm joins the All Japan Security Service Association (AJSSA) and employs technicians with professional expertise. Based on this, it is appropriate to make up above three organizations in AJSSA and send external experts on the Guideline Development Group and the Systematic Review Team for the wireless HS's guideline development.

\section{B. Scope \\ 1) In medical guidelines}

Scope is a document created by the Guideline Development Group and it contains the range of guidelines, details of the systematic review, and the schedule for the guideline publishing.

\section{2) In wireless $H S$ guidelines}

First of all, the Guideline Development Group defines the range of guidelines and cites the three problems (False Alarms, SIGINT, and Alarm Failure) to solve. Next, in each problem, this group should set a question which is discussed by the Systematic Review Team. For example, in the false alarm, "What kind of technologies are there to solve the false alarm?" is set. In addition, this group creates the evaluation forms used in the process of systematic review and defines the strength of the recommendation based on the said form. For example, the following descriptions are considered: "Recommend to adopt (strong)", "Recommend to adopt with condition (medium)", and "Recommend not to adopt (weak)."

\section{Systematic Review}

\section{1) In medical guidelines}

After receiving the questions from the scope process, Systematic Review Team answers each question and provides the level of recommendation by using the evaluation form. In order to ensure the fairness of evaluation, created evaluation forms undergoes pre-evaluation done by outside experts.

\section{2) In wireless HS guidelines}

Concrete methods which are proposed in academic journals and described from section 2 to 3 in this paper will become the intended items for review. It is desirable for the created evaluation forms to undergo evaluation by experts who are not affiliated with the security service firm or manufacturer (e.g., government researcher).

\section{Recommendation}

\section{1) In medical guidelines}

Guideline Development Group determines the strength of recommendation based on the evaluation forms created by the Systematic Review Team. At this time, this group places importance on the balance between advantages (cure/recovery) and drawbacks (costs, side effects, and operative scar) for patients. 


\section{2) In wireless HS guidelines}

Guideline Development Group should evaluate the technologies carefully from the following viewpoints: (1) the balance between merits (preventing damages from intrusion) and demerits (initial and running costs) for household members who use wireless HS daily, and (2) the balance between advantages (revenue) and disadvantages (development and operational costs) for security service firms which provides the wireless HS.

\section{E. Finalization}

\section{1) In medical guidelines}

Guideline Development Group compiles the documents created by each process from preparation to recommendation and makes the draft of guidelines. After the evaluation by the outside experts, the draft is approved by the Guideline Commissioning Board. Maintenance method after publication (e.g., organizational frameworks, evaluation of effectiveness, and judgment standard for revision) are described on the guidelines.

\section{2) In wireless HS guideline}

Guideline Development Group conducts the same procedures described above. In draft evaluation, it is desirable to undergo evaluation by experts from government institutes or universities same as in section 4.3.

\section{F. After Publication}

\section{1) In medical guidelines}

With the view to promote using the developed guidelines on the medical ground, printed and digital version are published by the Guideline Commissioning Board. Practical version (for daily medical care), simplified version (for quick reference), and general version (for patient and its family) are also published. These versions are revised every three or four years.

\section{2) In wireless $H S$ guidelines}

Guideline Commissioning Board conducts the same procedures described above. However, developing and publishing the general version of guidelines (for household member) is unnecessary because unlike the medical guidelines, this guidelines are not directly related to people's life and death. However, if the Guideline Commissioning Board is asked to present all guidelines by the household member, showing/giving practical or simplified version will do. This can also help saving cost for the development and publication of HS guidelines.

\section{CONCLUSIONS}

In order to resolve the three problems (False Alarm, SIGINT, and Alarm Failure) on the wireless HS, the author has developed and showed the example solutions and verified its effectiveness for practical use. These solutions improve the security of wireless communication and the reachability of alarm signals while saving sensor's battery power, which is less than or equal to the current consumption level. Furthermore, author has discussed the guidelines development and its expansion which are referred by security service companies and manufacturers during developing wireless HS. The vulnerability of wireless HS could cause damages not only household member but also the human infrastructure like police and security service companies. Due to this, the author intends to minimize its impact by sharing technical knowledge throughout the guidelines.

In order to verify the communication protocols' effectiveness, the author will implement above protocols on the prototype and measure the sensor's electric consumption. The Author also proposes the development and expansion of the wireless HS's guidelines to All Japan Security Service Association (AJSSA) which composed of the companies providing home security service. In addition, the author is considering to contribute the wireless HS's quality improvement by introducing Japanese technologies to international conferences on security technologies.

\section{REFERENCES}

[1] L. Lamb. Home insecurity: No alarms, false alarms, and SIGINT. [Online].

Available: https://media.defcon.org/DEF\%20CON\%2022/DEF\%20CON\%2022 $\% 20$ presentations/Logan\%20Lamb/DEFCON-22-Logan-Lamb-HOM E-INSECURITY-NO-ALARMS-FALSE-ALARMS-AND-SIGINTWP.pdf

[2] B. Rhodes, "ADT sued, claimed 'easily hacked'," IPVM, Nov. 17 2014.

[3] P. Lioio, "Understanding EMI noise in power-system design," Electronic Design, Apr. 24, 2012.

[4] Radio-Electronics.com. Multipath fading. [Online]. Available: http://www.radio-electronics.com/info/propagation/multipath/multipat h-fading.php

[5] N. Fujita. Knowing 920MHz band (Japanese text). [Online]. Available: http://www.kumikomi.net/archives/2014/06/rohm_920_mhz.php

[6] ROHM Semiconductor GmbH. ROHM-Lapis sub-GHz RF products for smart metering applications. [Online]. Available: http://www.symmetron.ru/suppliers/lighting/files/lighting/components /wireless/Rohm/Products_for_SUB-GHZ.pdf

[7] Symmetric key encryption performance analysis. [Online]. Available: http://cs.uccs.edu/ cs591/studentproj/projF2008/

[8] C. Paar and J. Pelzl, Understanding Cryptography, Springer-Verlag, p. 31.

[9] E. Barker et al., Recommendation for Key Management - Part 1: General (Revision 3), NIST SP800-57, 2012.

[10] Evidence-Based Medicine, BMJ Publishing Group Ltd, December 2014, vol. 19, issue 6 .

[11] Japan Council for Quality Health Care, Minds: Medical Treatment Guideline Preparation Manual, July 2014.

[12] Agency for Healthcare Research and Quality, National Guideline Clearinghouse (NGC).

[13] National Institute for Health and Care Excellence (NICE). [Online]. Available: http://www.nice.org.uk/

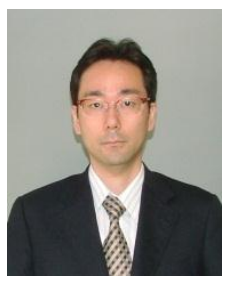

Masaki Fujikawa was born on February 6, 1974 in Tokushima Prefecture, Japan. He received his master's degree in information engineering in 1998 from Tokushima University, Tokushima Prefecture Japan. In 2004, he received his Ph.D. degree in information engineering from Chuo University, Tokyo, Japan.

He joined security service company ALSOK, in Tokyo Japan in 1998. From October 2002 to March 2011, he doubled as a guest researcher of the Research and Development Initiative, Chuo University. From November 2010 to Mach 2012, he was the director of an information security R\&D project of METI (Ministry of Economy, Trade and Industry, Japan). Now, he is one of the chief researchers of his company, and researching on different security and safety systems in order to build a safer society.

Dr. Fujikawa is a member of IEEE, IPSJ and the steering committee of the IPSJ Computer Security Group. Dr. Fujikawa received the best paper and specially selected paper awards from IPSJ and best demonstration award from the 4th IFIP WG 11.11 International Conference on Trust Management in 2010 . 